\title{
Déterminants de la gouvernance forestière dans les forêts communautaires de l’Est-Cameroun
}

\section{Rodrigue FAPA NANFACK ${ }^{1,2}$ \\ NanCy GÉLINAS ${ }^{1}$ \\ Kadiri Serge Вово ${ }^{2}$ \\ Hugo AsSELIN ${ }^{3}$ \\ François Hiol Hiol ${ }^{2}$ \\ Carine Atole NtATSOULA ${ }^{2}$}

\section{${ }^{1}$ Université Laval}

Faculté de foresterie, géographie et géomatique

2405 rue de la Terrasse

Québec (Québec) G1V 0A6

Canada

2 Université de Dschang

Faculté d'agronomie et des sciences agricoles

Département de foresterie

BP 222, Dschang

Cameroun

${ }^{3}$ Université du Québec en Abitibi-Témiscamingue École d'études autochtones 445 boulevard de l'Université Rouyn-Noranda (Québec) J9X 5E4 Canada

\section{Auteur correspondant /}

Corresponding author:

Rodrigue Fapa Nanfack -

rodrigue.fapa-nanfack.1@ulaval.ca

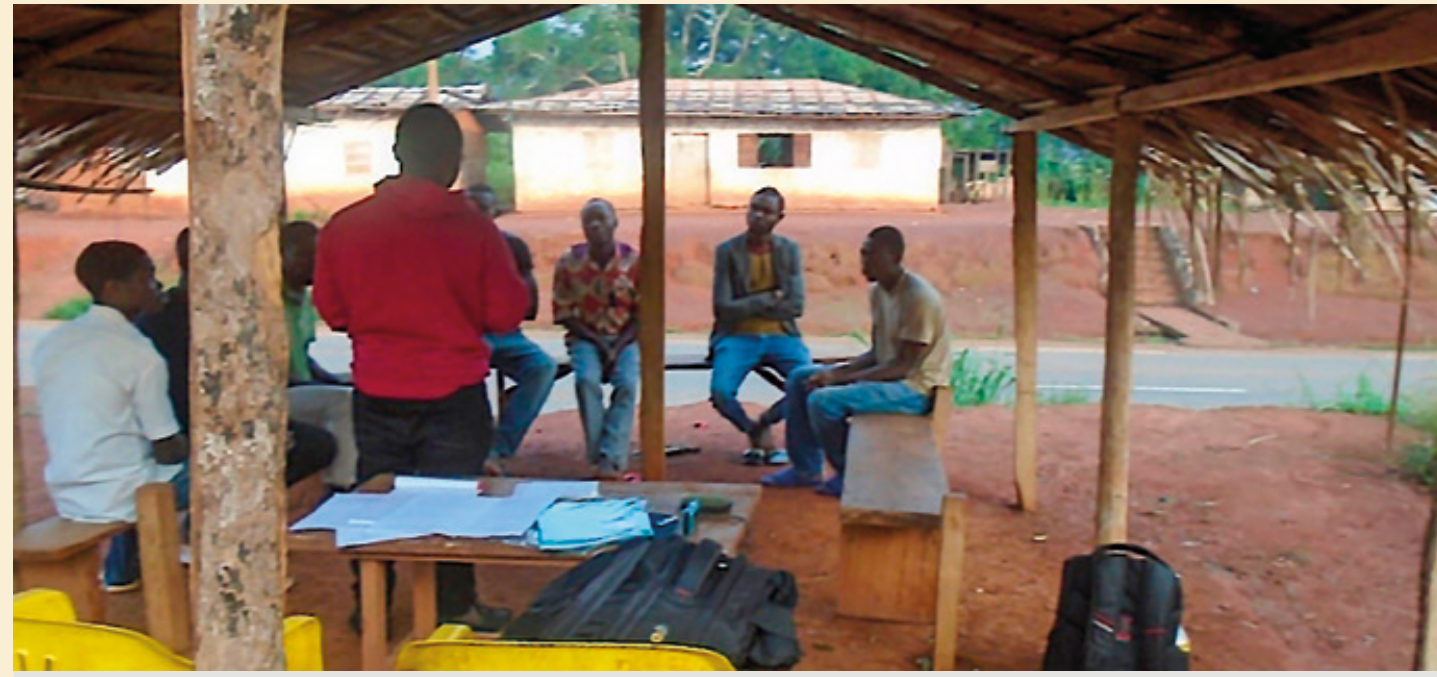

Photo 1.

Séance d'analyse de la gouvernance locale avec un groupe de discussion composé des représentants des divers intérêts (gestionnaires de FC, pouvoir traditionnel, jeunes et femmes) pour la forêt communautaire. Photo. R. Fapa Nanfack.

Doi : 10.19182/bft2020.343.a31713 - Droit d'auteur (c) 2020, Bois et Forêts des Tropiques - (c) Cirad - Date de soumission : 8 avril 2019 ; date d'acceptation : $1^{\text {er }}$ octobre 2019 ; date de publication : 15 janvier 2020.
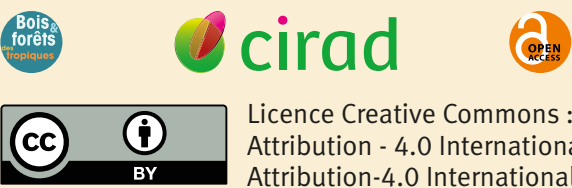

Citer l'article / To cite the article

R. Fapa Nanfack, N. Gélinas, K. S. Bobo, H. Asselin, F. Hiol Hiol, C. Atole Ntatsoula, 2019. Déterminants de la gouvernance forestière dans les forêts communautaires de l'Est-Cameroun. Bois et Forêts des Tropiques, 343 : 53-66. Doi : https://doi.org/10.19182/bft2020.343.a31713 


\section{RÉSUMÉ}

\author{
Déterminants de la gouvernance \\ forestière dans les forêts \\ communautaires de l'Est-Cameroun
}

Au Cameroun, les communautés locales font face à des défis de gouvernance forestière et de propriété des forêts communautaires. Les déterminants du succès de la gouvernance forestière ont été étudiés dans douze forêts communautaires de l'Est-Cameroun. Des groupes de discussion et des entrevues individuelles ont permis de réaliser la cartographie des acteurs d'appui, de documenter la perception de la gouvernance des forêts communautaires et d'en identifier les déterminants, pour ensuite proposer une échelle de gouvernance des forêts communautaires. Les résultats montrent que les acteurs d'appui influencent grandement la création des forêts communautaires. L'exception de la FC A3 montre qu'une communauté peut créer une forêt communautaire sous la seule impulsion du chef traditionnel, sans soutien extérieur. La perception de la gouvernance forestière par les communautés était positive lorsque l'appui à la création de la forêt communautaire provenait d'acteurs tournés vers la communauté (organisations non gouvernementales, administration des forêts et Église) et négative lorsque l'appui provenait d'acteurs tournés vers la ressource ligneuse (élites et opérateurs forestiers privés). Le diagramme des déterminants relationnels entre communautés et acteurs d'appui, basé sur la cohésion entre les principes de gouvernance (participation, transparence, légitimité, équité, intégration, imputabilité, capacité et adaptabilité), met en évidence les actions correctives possibles pour mieux tenir compte des besoins des communautés afin d'atteindre la dévolution sur l'échelle de la gouvernance.

Mots-clés : communautés locales, gestion forestière, dévolution, participation, transparence, légitimité, équité, intégration, Cameroun.
R. Fapa Nanfack, N. GÉlinas, K. S. Bobo, H. Asselin, F. Hiol Hiol, C. Atole ntatsoula

\section{ABSTRACT}

\section{Determinants of forest governance in community forests of East Cameroon}

In Cameroon, communities face challenges related to governance and ownership of community forests. The determinants of governance were studied in 12 community forests of East Cameroon. Focus group discussions and one-on-one interviews were used to identify supporting groups, identify perceptions and determinants of governance, and develop a governance scale for community forests. Results show that support groups greatly influence the creation of community forests. The exception of FC A3 shows that a community can create a community forest under the sole impulsion of traditional chief, without external support. The perception of governance by communities was positive in community forests created with the support of groups with interests in the community (non-governmental organisations, forest authorities, Church), and negative when support came from groups interested in wood production (elites, private forest operators). The diagram of relations between communities and support groups based on cohesion between governance principles (participation, transparency, legitimity, equity, integration, accountability, capacity and adaptability), highlights potential corrective actions to reach the devolution level on the scale of governance.

Keywords: local communities, forest management, devolution, participation, transparency, legitimacy, fairness, integration, Cameroon.

\section{RESUMEN}

\author{
Determinantes de la gobernanza forestal \\ en los bosques comunitarios \\ del Camerún oriental
}

En Camerún, las comunidades locales enfrentan desafíos en la gobernanza forestal y la propiedad forestal comunitaria. Se estudiaron los determinantes de la gobernanza forestal exitosa en doce bosques comunitarios del Camerún oriental. Los grupos focales y las entrevistas individuales permitieron mapear a los actores de apoyo, documentar la percepción de la gobernanza forestal comunitaria e identificar sus determinantes, para luego proponer una escala de gobernanza forestal comunitaria. Los resultados muestran que los actores de apoyo tienen una influencia significativa en la creación de bosques comunitarios. La excepción del A3 CF muestra que una comunidad puede crear un bosque comunitario únicamente a instigación del jefe tradicional, sin apoyo externo. La percepción de la gobernanza forestal por parte de las comunidades fue positiva cuando el apoyo a la creación de bosques comunitarios provino de actores orientados a la comunidad (organizaciones no gubernamentales, administración forestal y la Iglesia) y negativa cuando el apoyo provino de actores orientados a los recursos forestales (élites y operadores forestales privados). El diagrama de determinantes relacionales entre comunidades y actores de apoyo, basado en la cohesión entre los principios de gobernabilidad (participación, transparencia, legitimidad, legitimidad, equidad, equidad, integración, rendición de cuentas, capacidad y adaptabilidad), destaca las posibles acciones correctivas para tener mejor en cuenta las necesidades de las comunidades con el fin de lograr la devolución en la escala de gobernabilidad.

Palabras clave: comunidades locales, gestión forestal, devolución, participación, transparencia, legitimidad, equidad, integración, Camerún. 


\section{Introduction}

L'État du Cameroun, au lendemain de son indépendance et sous l'influence de la Banque mondiale (Brunner et Ekoko, 2000), a dû améliorer sa gouvernance forestière pour faire participer les communautés jusqu'alors exclues (Kouna Eloundou, 2012). L'État a ainsi promulgué la loi forestière de $1994^{1}$. L'article 20 de cette loi reconnaît deux domaines forestiers : non permanent et permanent. Le domaine forestier non permanent représente les terres forestières (coutumières) susceptibles d'être affectées à des utilisations autres que forestières, ce qui inclut les forêts communautaires (FC). L'article 37 (1) prévoit que l'administration des forêts doit accorder une assistance technique gratuite aux communautés villageoises qui manifestent l'intérêt de prendre en charge la gestion des ressources forestières.

Aujourd'hui, malgré la reconnaissance des bases théoriques des FC, leur application s'avère difficile (Julve Larrubia et al., 2007). La loi ne semble pas avoir permis d'accroître l'assistance aux FC pour en assurer la bonne gouvernance. Les agents forestiers manquent de formation en foresterie communautaire et exigent parfois des pots-de-vin auprès des communautés (Brown et Schreckenberg, 2001 ; Alemagi et Kozak, 2010). Dans les communautés, le faible niveau d'alphabétisation, le manque d'expérience des procédures bureaucratiques et la méfiance des chefs traditionnels envers les FC nuisent à la gouvernance forestière (CARFAD et MINFOF, 2006).

Les organisations non gouvernementales (ONG), les élites et les opérateurs forestiers privés ont été présentés aux communautés comme des acteurs d'appui à la création et à l'amélioration de la gouvernance des FC (Burnham, 2000 ; Lescuyer et al., 2016). Au Cameroun et dans d'autres pays en développement, ces acteurs ont financé la création de FC, donné des formations et entrepris des dons d'équipements forestiers aux communautés (Djeumo, 2001 ; Afroz et al., 2016). Cependant, certains de ces acteurs ont manipulé les communautés afin d'obtenir des résultats à court terme pour légitimer leurs actions d'accompagnateur ou de porte-parole des besoins des communautés sur le territoire pour se rendre incontournables et capter une partie significative des fonds destinés aux FC, au détriment des communautés (Lescuyer et al., 2019). Ces projets, favorisant les intérêts externes aux communautés, ont échoué. Cuny (2011) et Afroz et al. (2016) expliquent en partie cet échec par une planification descendante (top-down), donnant seulement l'illusion d'une participation des communautés à la gestion des forêts.

Pour permettre aux communautés de s'approprier les $\mathrm{FC}$ et d'en assurer la gestion, il s'avère nécessaire de comprendre la dynamique de gestion des FC, les rôles des acteurs d'appui à leur création et les défis de leur gouvernance. Notre objectif était d'identifier les déterminants de la gouvernance des FC de l'Est-Cameroun et d'en dégager une échelle de gouvernance, en fonction des acteurs d'appui. Cette échelle pourra être utilisée pour établir le diagnostic de la gouvernance dans les FC afin de permettre aux communautés de mieux se les approprier.

1. Loi n $94 / 01$ du 20 janvier 1994 portant sur le régime des forêts, de la faune et de la pêche. Ministère de l'Environnement et des Forêts. République du Cameroun.

\section{Gouvernance des forêts communautaires}

Cette étude s'inscrit dans le courant de pensée politique institutionnaliste de l'auto-organisation (Ostrom, 2010) pour expliquer les règles de fonctionnement de la gouvernance des FC. L'évaluation d'un système de gouvernance s'appuie sur des principes de participation, transparence, légitimité, équité, intégration, imputabilité, capacité et adaptabilité aux échelles nationale et locale (Lockwood et al., 2010).

\section{Principes de gouvernance des forêts communautaires}

Selon Lockwood et al. (2010), la participation renvoie à la possibilité pour les citoyens et les parties prenantes d'influencer et de s'impliquer dans la prise de décision et les actions en découlant. La participation implique la prise en compte de la variabilité des acteurs qui ont un intérêt dans le processus de décision, les considérant tous sur un pied d'égalité. La participation des parties prenantes à l'élaboration des politiques renforce la transparence, soit la visibilité du processus décisionnel, la clarté de communication du raisonnement sur lequel les décisions s'appuient et la disponibilité de l'information pertinente à propos de la performance organisationnelle (Lockwood et al., 2010). La transparence permet la mise en place d'un cadre juridique favorisant l'accès du public à l'information (Profor et FAO, 2011). La transparence permet de savoir qui a pris une décision et les conditions dans lesquelles cette décision a été prise. Dans les FC, le manque de transparence peut affecter négativement la légitimité, qui réfère à l'intégrité d'une organisation et à la validité de son autorité à gouverner (Lockwood et al., 2010). La légitimité peut être conférée par statut démocratique ou découler de l'acceptation par les parties prenantes du pouvoir de gouverner d'une organisation. Pour être légitime, la gouvernance doit s'opérer au plus bas niveau décisionnel efficace. La légitimité nécessite une reconnaissance minimale par les autorités externes du droit à l'auto-organisation (Ostrom, 2010).

L'équité renvoie au respect des points de vue des parties prenantes, à l'absence de biais dans le processus décisionnel et à la répartition des coûts et des bénéfices des décisions prises (Lockwood et al., 2010). L'équité doit tenir compte des aspects de répartition entre les différents groupes d'intervenants à un moment donné (répartition intragénérationnelle), ainsi que des aspects de répartition à travers le temps (répartition intergénérationnelle) pour une meilleure intégration des parties prenantes (Banque mondiale, 2009).

L'intégration réfère à la coordination entre les différents niveaux de gouvernance (intégration verticale) et entre les différentes organisations de gouvernance au même niveau (intégration horizontale) afin d'aligner les priorités, les plans d'action et les activités (Lockwood etal., 2010). L'imputabilité désigne l'acceptation de la responsabilité de décision et d'action (Lockwood et al., 2010). Elle nécessite un renforcement des capacités des parties prenantes à s'acquitter de leurs responsabilités. Les capacités s'appuient sur des systèmes, des plans, des ressources, du leadership, des connaissances et de l'expérience (Lockwood et al., 2010). Le développement 
des capacités renforce l'adaptabilité, c'est-à-dire la capacité de l'organisation à réorganiser ses processus en réponse à des conditions changeantes. Pour ce faire, il faut intégrer les nouvelles connaissances, anticiper les risques, les menaces et les opportunités (Lockwood et al., 2010).

\section{Tenure foncière et forêts communautaires au Cameroun}

Avant les années 1990, la gestion foncière au Cameroun a été marquée par une centralisation de la gestion des terres et des forêts par l'État. Sous la contrainte des programmes d'ajustement structurel ${ }^{2}$ de la Banque mondiale en 1989 et au lendemain du sommet de Rio en 1992, des réformes de décentralisation ont visé à améliorer la participation des communautés à la prise de décision (Karsenty, 2010, 2017). Le processus de réforme des FC était au départ ambitieux afin de permettre aux communautés d'exploiter les forêts du domaine permanent. Ce processus a été soutenu par des réformateurs nationaux et combattu par différents groupes de pression, mais également par certains fonctionnaires du ministère des Forêts. Cependant, du fait de la complexité du contexte politique des années 1990, les ambitions ont dû être revues à la baisse et les FC ont été classées dans le domaine non permanent. Ainsi, seule une partie des réformes projetées a pu être mise en œuvre (Brunner et Ekoko, 2000 ; Karsenty, 2017).

Malgré la volonté de décentraliser la gestion forestière, l'État, dans la loi forestière de 1994, n'a pas reconnu le rôle des chefs traditionnels dans la gestion des forêts (Oyono, 2005 ; Alemagi et Kozak, 2010). Le processus de décentralisation a été structuré sur un socle juridique dualiste, où un dispositif légal de droit moderne se superpose à un dispositif de droit coutumier, le premier tendant à ignorer le second (Ongolo et Brimont, 2015). Dans ce contexte, l'État a tenté de contourner les chefs traditionnels en mettant en place les FC. Dès lors, certains chefs traditionnels ont parfois encouragé l'exploitation illégale, ce qui nuisait à la gouvernance forestière et désavouait les gestionnaires des FC (Oyono, 2005 ; Alemagi et Kozak, 2010). Le manque d'harmonisation a ainsi créé une concurrence entre l'État et les chefs traditionnels (Karsenty, 2017 ; Samndong et Vatn, 2018).

La création des FC peut être demandée par les communautés qui exercent leurs activités dans une forêt de moins de 5000 hectares, dans le respect des règles d'un plan de gestion simple approuvé par l'administration des forêts (Julve Larrubia et al., 2013 ; Lescuyer et al., 2019 ; Minang et al., 2019). La convention de gestion d'une FC ne confère à la communauté ni les droits de propriété sur la terre, ni quelque titre de propriété que ce soit sur la forêt elle-même. L'État limite ainsi les communautés au droit d'usufruit, de collecte des produits forestiers non ligneux (PFNL) et à la commercialisation du bois issu des FC (Bigombe Logo, 2007). Pour bénéficier d'une FC, l'État exige que les dirigeants de la communauté se regroupent en entité juridique de gestion (groupe d'initiative commune, groupe d'intérêt économique, coopérative ou association) puisque les institutions villageoises ne sont pas reconnues comme des entités juridiques (Ezzine de Blas et al., 2009). Plusieurs communautés se regroupent parfois sous une même entité juridique, mettant en commun leurs territoires pour contourner l'obstacle d'insuffisance d'espace de forêt exploitable (Milol, 2000). Les communautés peuvent théoriquement exploiter ces forêts en régie ou les confier en sous-traitance. Or, la réalité observée sur le terrain est que la plupart des FC, à l'exemple des FC initiées par les élites, les chefs traditionnels et les opérateurs forestiers privés, sont exploitées en sous-traitance par les acteurs d'appui à cause des coûts élevés et de la lourdeur de la démarche administrative (Julve Larrubia et al., 2013).

\section{Acteurs d'appui aux forêts communautaires}

Les acteurs d'appui ont une influence directe ou indirecte sur les FC. Au Cameroun, la majorité des dossiers d'attribution des FC ont bénéficié de l'appui des élites (individus ayant un statut supérieur en raison des caractéristiques économiques et éducatives), des ONG ou des opérateurs forestiers (Bigombe Logo, 2007 ; Lescuyer et al., 2016). Une étude de Ezzine de Blas et al. (2009) mentionne qu'au Cameroun les ONG se sont intéressées aux FC de petite superficie avec peu d'espèces de valeur, contrairement aux opérateurs forestiers qui ont choisi des FC de grande superficie. Les ONG étaient des acteurs tournés vers la communauté et favorisaient le renforcement des capacités (Djeumo, 2001). Quant aux élites et aux opérateurs forestiers, ils étaient des acteurs tournés vers la ressource ligneuse et limitaient leur coopération aux procédures administratives d'obtention des FC.

\section{Méthodes}

\section{Site d'étude}

Dans la région de l'Est-Cameroun, d'une superficie de 109002 km², les FC étudiées représentent $335 \mathrm{~km}^{2}$. Le climat y est de type équatorial guinéen, les précipitations sont de $1500-2000 \mathrm{~mm}$ par an et la température moyenne annuelle est de $23{ }^{\circ} \mathrm{C}$ (PNDP, 2012). Cette région a bénéficié du programme gouvernemental pour le Renforcement des initiatives de gestion communautaire (RIGC) qui visait à accompagner les communautés à travers ses volets équipement, formation et rédaction des plans simples de gestion dans les FC.

Suivant une méthode non probabiliste, 12 FC ont été retenues, sur environ 119 FC présentes dans la zone d'étude, en raison de leur facilité d'accès, de la diversité des acteurs d'appui, de la disponibilité d'informations sur la légalité et la traçabilité du bois, et en lien avec une étude concomitante de Fapa Nanfack et al. (données non publiées) (tableau I ; figure 1). L'agriculture est l'activité la plus pratiquée dans la zone. Les principales cultures sont le manioc (Manihot esculenta) et le maïs (Zea mays). Les acheteurs du bois des FC sont des opérateurs forestiers originaires du Nord-Cameroun.

\section{Groupes de discussion et entretiens individuels semi-dirigés}

L'étude a été menée de mai à octobre 2017. Des groupes de discussion ont été constitués dans chacune des FC, composés de 6 à 10 personnes représentant divers intérêts (gestionnaires de FC, pouvoir traditionnel, jeunes et femmes). Le choix des participants dans chaque groupe

2. Aide financière et réformes économiques imposées 
Tableau I.

Caractéristiques des forêts communautaires (FC) étudiées dans l’Est-Cameroun.

\begin{tabular}{|c|c|c|c|c|c|c|}
\hline $\begin{array}{l}\text { Forêt } \\
\text { communautaire }\end{array}$ & Département & $\begin{array}{l}\text { Année de } \\
\text { création } \\
\text { de l'entité }\end{array}$ & $\begin{array}{c}\text { Année } \\
\text { d'obtention } \\
\text { de la FC }\end{array}$ & $\begin{array}{c}\text { Première } \\
\text { exploitation }\end{array}$ & $\begin{array}{l}\text { Superficie } \\
\text { (ha) }\end{array}$ & Initiateur(s) \\
\hline A1 & Haut-Nyong & 1998 & 2000 & 2016 & 2300 & Partenariat ONG internationale et ONG locale \\
\hline $\mathrm{A} 2$ & Haut-Nyong & 1999 & 2008 & 2008 & 2970 & Administration des forêts \\
\hline A3 & Haut-Nyong & 2001 & 2009 & 2011 & 3900 & Chefs traditionnels \\
\hline A4 & Haut-Nyong & 2012 & 2015 & 2015 & 2300 & Opérateur forestier privé \\
\hline A5 & Haut-Nyong & 2014 & 2016 & 2016 & 4760 & Opérateur forestier privé \\
\hline A6 & Haut-Nyong & 1998 & 2001 & 2009 & 4000 & Élite \\
\hline A7 & Kadey & 2007 & 2009 & 2010 & 1380 & Partenariat Église et ONG nationale \\
\hline A8 & Kadey & 2007 & 2010 & 2010 & 1931 & Partenariat Église et ONG nationale \\
\hline A9 & Kadey & 2007 & 2009 & 2011 & 1250 & Partenariat Église et ONG nationale \\
\hline $\mathrm{A} 10$ & Kadey & 1999 & 2007 & 2010 & 2365 & Élite \\
\hline A11 & Kadey & 2014 & 2015 & 2015 & 2645,5 & Élite \\
\hline A12 & Kadey & 2015 & 2016 & 2016 & 3730 & Élite \\
\hline
\end{tabular}

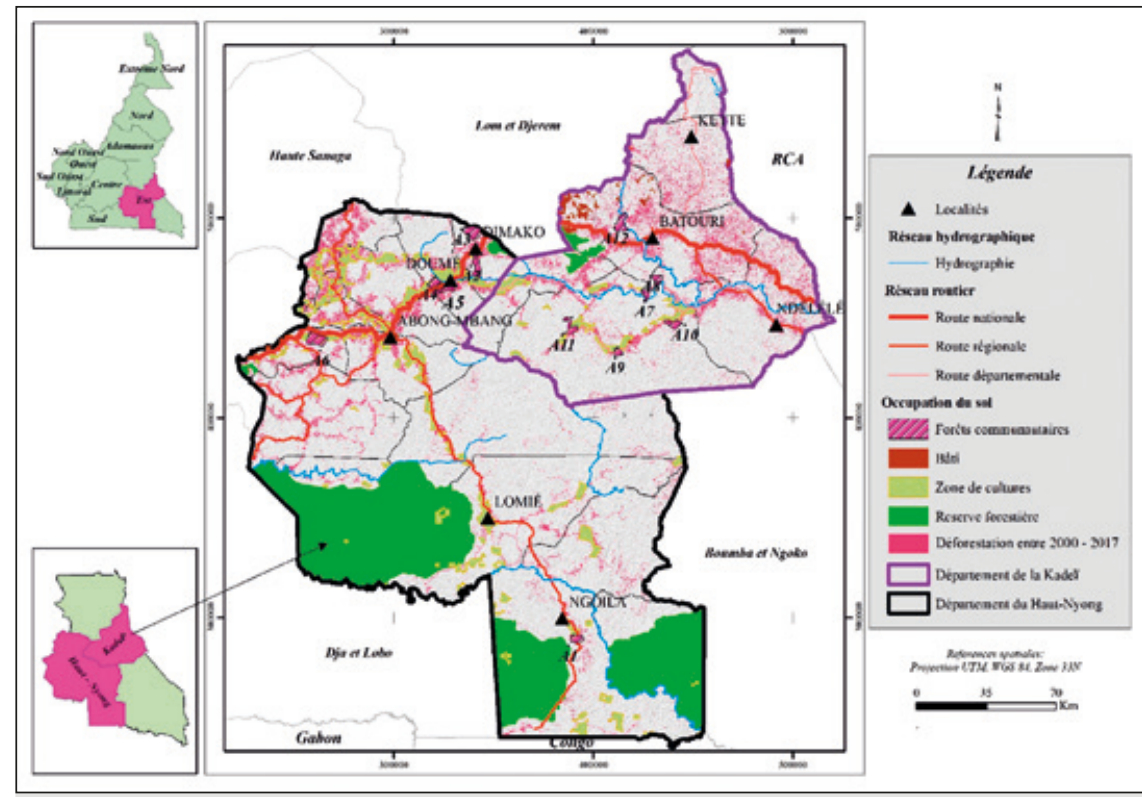

Figure 1.

Localisation des forêts communautaires (FC) étudiées dans l'Est-Cameroun.

Source : fichiers cartographiques de l'INC (2014).

Des entretiens individuels semidirigés ont été réalisés avec 20 personnes-ressources. La sélection des personnes-ressources s'est faite par « boule de neige» (Beaud, 2009), en commençant par les ONG nationales pour s'étendre à l'administration des forêts, aux organisations de recherche et aux exploitants forestiers. Le guide d'entrevue portait sur les forces et les faiblesses de la gouvernance des FC. Les groupes de discussion et les entretiens individuels avaient une durée moyenne d'environ $1 \mathrm{~h}$.

\section{Analyse des données}

L'analyse thématique des données a été facilitée par l'utilisation du logiciel NVivo (QSR International Inc.). Les transcriptions des groupes de discussion et des entretiens individuels ont été codées selon différents thèmes (Paillé et Mucchielli, 2012). Bien que l'utilisation des groupes de discussion puisse constituer un biais, cette méthode a néanmoins été retenue, car l'étude ne visait pas à documenter les conflits internes dans les communautés, ni à présenter un

s'est fait par « choix raisonné » (Geoffrion, 2009). Seules les personnes ayant vécu le processus de création de la FC ou connaissant le fonctionnement de la FC ont été sélectionnées avec l'aide des gestionnaires de FC. Les groupes de discussion ont été animés à l'aide d'un guide d'entretien axé sur les thématiques de l'historique de création des FC, des acteurs d'appui et des principes de gouvernance (tableau II). échantillon statistiquement représentatif des communautés. L'étude visait les dynamiques entre les FC et les acteurs d'appui. La triangulation utilisée pour analyser les données a permis d'augmenter la validité des résultats. La diversité des participants aux groupes de discussion (présence de Bakas et de femmes venues avec leur mari) a aussi permis de réduire les biais. 
Tableau II.

Thèmes abordés afin de documenter les principes de gouvernance des forêts communautaires (FC) (d'après Banque mondiale, 2009 ; Lockwood et al., 2010 ; Ostrom, 2010).

\begin{tabular}{ll}
$\begin{array}{l}\text { Principes de } \\
\text { gouvernance }\end{array}$ & Thèmes abordés \\
\hline Légitimité & Processus de désignation des gestionnaires de la FC \\
\hline Participation & Implication des communautés dans la prise de décision de manière formelle et informelle \\
\hline Transparence & Visibilité du processus et diffusion de l’information \\
\hline Imputabilité & Processus de responsabilité pour la reddition de compte dans la FC \\
\hline Équité & Processus de répartition des retombées financières de la FC \\
\hline Capacité & Compétences et expertises acquises par les communautés pour la gestion \\
\hline Intégration & Liens entre les différents acteurs \\
\hline Adaptabilité & Réorganisation des communautés pour faire face aux changements \\
\hline
\end{tabular}

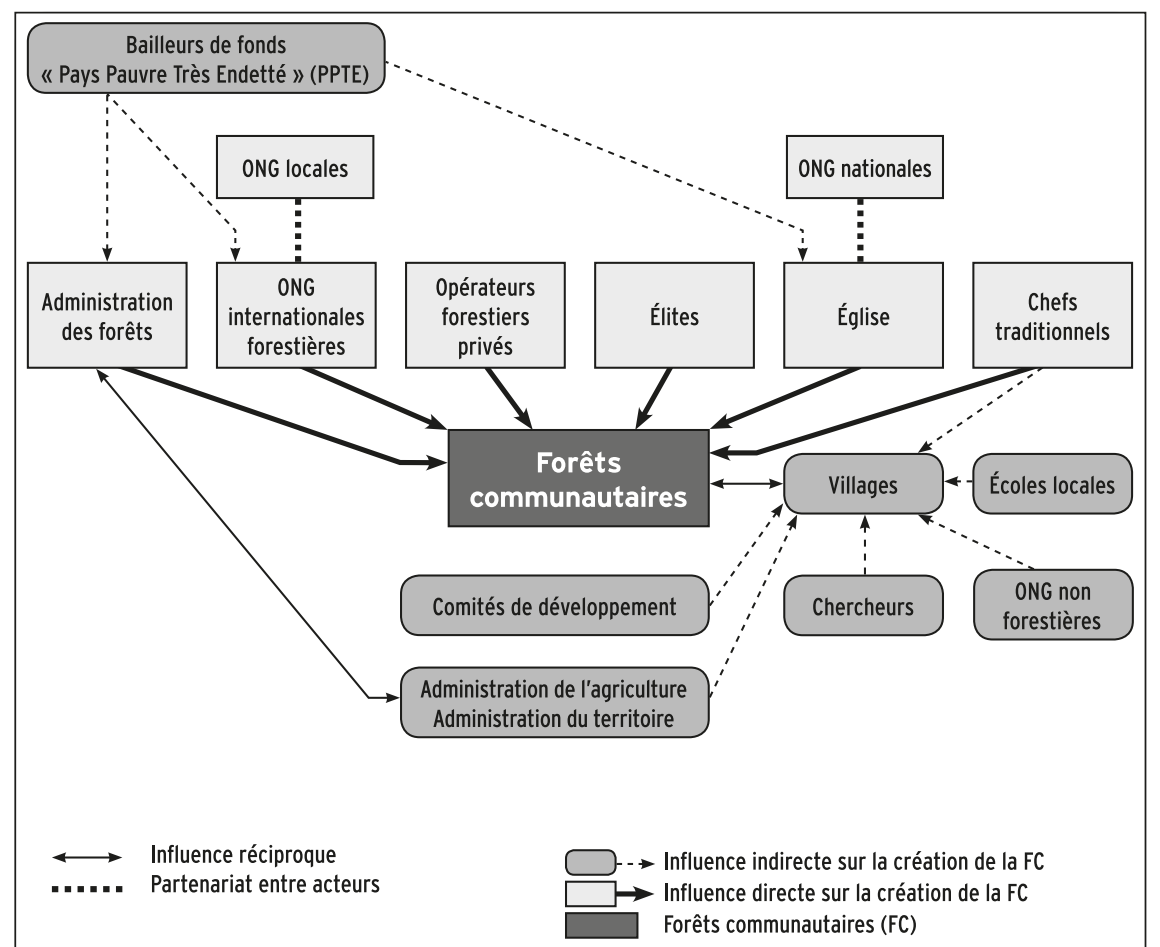

Figure 2.

Cartographie des acteurs d'appui aux forêts communautaires (Est-Cameroun). de la gouvernance des FC a été élaboré en s'inspirant du diagramme des relations causales entre les divers facteurs de succès de la FC établi par Baynes et al. (2015). Les déterminants ont été obtenus sur la base des informations récurrentes ressorties des entrevues et des groupes de discussion. Finalement, à partir de l'ensemble de ces informations, une échelle de gouvernance des FC a été élaborée, inspirée de l'échelle de participation de Arnstein (1969) et de l'échelle des niveaux de cogestion de Rodon (2003). Les acteurs d'appui ont été situés sur cette échelle en fonction de leur niveau de respect des principes de gouvernance.

\section{Résultats}

\section{Cartographie des acteurs d'appui et leurs apports}

Les résultats permettent de classer les acteurs d'appui à la création des FC en deux catégories, soit avec influence directe ou indirecte (figure 2). Les interactions mutuelles entre les acteurs d'appui, à l'exemple de celles entre l'administration des forêts et les ONG, n'ont pas été prises en compte dans cette cartographie.
Dans un premier temps, les résultats ont permis de cartographier les acteurs d'appui et leurs liens avec les communautés. Dans un deuxième temps, la conformité de l'approche des acteurs d'appui en respect des principes de gouvernance de Lockwood et al. (2010) a été classée selon les catégories "Respect complet », "Respect partiel » ou « Non-respect ». Le classement s'est effectué en fonction du nombre de principes respectés. Dans un troisième temps, un diagramme des déterminants positifs et négatifs
Influence directe sur la création des forêts communautaires Plusieurs groupes d'acteurs ont influencé directement la création des FC: les ONG internationales et leurs représentants locaux, l'administration des forêts, l'Église et les ONG nationales partenaires. Selon une personne-ressource (P1), ces acteurs pouvaient contrôler jusqu'à 80 \% du processus de création des FC. Ces acteurs organisaient des formations sur le rôle des communautés dans la FC, réalisaient des inventaires forestiers, finançaient et suivaient les dossiers 
de création des FC. Ils réservaient des fonds provenant de l'initiative " Pays pauvre très endetté » (PPTE) pour la mise en œuvre de projets de FC. De leur côté, les communautés contribuaient en intervenant dans les activités d'inventaire comme machetteurs.

Les ONG internationales s'appuyaient sur des ONG locales dans le cadre des partenariats pour promouvoir les FC. Dans la FC A1, l'ONG internationale et son représentant local ont organisé des formations sur la législation forestière et sur l'utilisation de scieries mobiles (lucas mills). La FC A3 a été initiée par les chefs traditionnels de deux villages. Les chefs, n'ayant pas les moyens de financer la création de la FC, ont exigé des cotisations de la part des communautés. Les élites et les opérateurs forestiers privés ont investi leurs propres fonds pour financer la création de FC et ont obtenu en échange l'exclusivité de l'exploitation du bois. Ces acteurs n'organisaient aucune formation.

\section{Influence indirecte sur la création des forêts communautaires}

L'administration de l'agriculture et l'administration du territoire intervenaient au quotidien en appuyant les communautés par leurs actions vers l'amélioration de la gouvernance dans les villages. Les différentes administrations entretenaient des relations de collaboration pour améliorer la gouvernance. Les chercheurs, les écoles locales et les ONG à vocation non forestière jouaient un rôle dans la sensibilisation et la formation des communautés. Les comités de développement définissaient les projets prioritaires pour les villages. Ces actions n'avaient aucune influence sur la création de la FC, mais elles en influençaient le fonctionnement. Les gestionnaires de FC vivaient dans les villages et participaient aux activités de formation et de sensibilisation.

Tableau III.

Caractérisation de la gouvernance forestière au sein des forêts communautaires (FC) étudiées en fonction des acteurs d'appui et du niveau de respect des principes de gouvernance.

Acteurs tournés vers la communauté

\begin{tabular}{|c|c|}
\hline Principes & $\begin{array}{l}\text { ONG internationale } \\
\text { et locale }\end{array}$ \\
\hline Participation & $\begin{array}{l}\text { Représentation de } \\
\text { toutes les couches } \\
\text { sociales dans la FC }\end{array}$ \\
\hline Transparence & $\begin{array}{l}\text { Disponibilité de } \\
\text { l'information écrite } \\
\text { et verbale }\end{array}$ \\
\hline Légitimité & $\begin{array}{l}\text { Vote des hommes et } \\
\text { nomination des } \\
\text { groupes marginalisés } \\
\text { (femmes et baka) }\end{array}$ \\
\hline Équité & $\begin{array}{l}\text { Réalisation et partage } \\
\text { des denrées }\end{array}$ \\
\hline Intégration & $\begin{array}{l}\text { Création de nouvelles } \\
\text { activités pour intégrer } \\
\text { les communautés }\end{array}$ \\
\hline Imputabilité & $\begin{array}{l}\text { ONG et communauté } \\
\text { suivent les documents } \\
\text { et activités de la FC }\end{array}$ \\
\hline Capacité & $\begin{array}{l}\text { Formation de toute } \\
\text { la communauté } \\
\text { pour améliorer } \\
\text { les capacités }\end{array}$ \\
\hline Adaptabilité & $\begin{array}{l}\text { Méfiance des } \\
\text { communautés } \\
\text { à cause des } \\
\text { contraintes de coûts }\end{array}$ \\
\hline
\end{tabular}

Église et ONG nationale

Représentation de toutes les couches sociales dans la FC

Disponibilité de l'information écrite et verbale

Identification du leader et nomination

des groupes

marginalisés (femmes et baka)

Réalisation et partage des denrées

Pas de création

d'activités hors du projet

Église et communauté suivent les documents et activités de la FC

Formation des gestionnaires pour améliorer les capacités

Méfiance des communautés à cause des contraintes de coûts
Administration
des forêts

Représentation de toutes les couches sociales dans la FC

Disponibilité de l'information écrite et verbale

\section{Identification du}

leader et nomination

des groupes

marginalisés

(femmes et baka)

Réalisation et partage des denrées

Pas de création

d'activités hors

du projet

Agent se charge du

suivi des documents de la FC

\section{Formation}

des gestionnaires

pour améliorer

les capacités

Méfiance des

communautés

à cause des

contraintes de coûts

Acteurs tournés vers la ressource ligneuse

Chefs traditionnels

Représentation

limitée à un groupe

restreint d'hommes

Disponibilité de

l'information écrite

et verbale

Désignation des

membres de la FC

par les chefs

Réalisation et partage des denrées

Activités se font indépendamment des autres acteurs

Chef suit le dossier de FC et opérateur forestier privé l'activité en forêt

Capacité s'acquiert

en côtoyant

les forestiers

Réceptifs

au changement

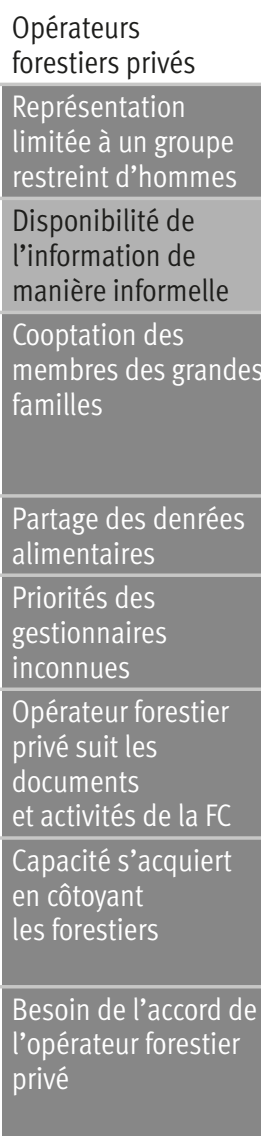

Élites

Représentation limitée à l'élite

Information inexistante Cooptation des membres du bureau de la FC par l'élite

Partage des denrées alimentaires

Priorités du

gestionnaire

inconnues

Opérateur forestier privé et élite suivent les dossiers et activités de la FC Capacité s'acquiert en côtoyant les forestiers Élites défavorables 


\section{Perception de la gouvernance des forêts communautaires en fonction des acteurs d'appui}

Un deuxième niveau de catégorisation permet de diviser les acteurs ayant une influence directe sur la création des FC en deux sous-groupes, à savoir les acteurs davantage tournés vers la communauté et ceux davantage tournés vers la ressource ligneuse. Les ONG internationales et leurs ONG locales partenaires, l'administration des forêts, l'Église et son ONG nationale partenaire sont des acteurs tournés vers la communauté. Quant aux élites, chefs traditionnels et opérateurs forestiers privés, ils sont des acteurs tournés vers la ressource ligneuse (tableau III). La perception de l'atteinte ou non des principes de bonne gouvernance forestière variait selon les deux groupes d'acteurs d'appui.

\section{Participation}

Dans les FC initiées par les acteurs tournés vers la communauté, la participation de tous les membres se faisait lors des assemblées générales. Dans la FC A1, l'ONG internationale et son partenaire local s'assuraient de l'implication de toute la communauté dans les activités et exigeaient la représentation sociale des couches marginalisées. Les femmes coordonnaient les activités liées aux PFNL et les hommes les activités d'exploitation du bois.

Les opérateurs forestiers privés, les chefs traditionnels et les élites ont plutôt favorisé l'exclusion des femmes et des jeunes de la gouvernance des FC. La participation à la création et la gestion de ces FC ont été limitées à un groupe restreint de leaders communautaires, d'élites, de chefs traditionnels ou d'opérateurs forestiers privés. Dans la FC A6, par exemple, les réunions étaient organisées hors du village et au domicile de l'élite où seuls les membres du bureau pouvaient participer. Les communautés n'appréciaient pas cette façon de faire.

\section{Transparence}

Dans les FC initiées par les acteurs tournés vers la communauté, les gestionnaires des FC mettaient l'information écrite et verbale à la disposition de la communauté afin d'assurer la transparence du processus décisionnel. Ces acteurs, ainsi que les chefs traditionnels, présentaient et lisaient les documents signés aux communautés. Les communautés appréciaient cette transparence. Dans la FC A3, par exemple, les documents ont été présentés par les chefs traditionnels pour justifier l'utilisation de l'argent cotisé par la communauté.

Dans les FC initiées par les élites et les opérateurs forestiers privés, la transparence était presque inexistante. La communauté n'avait jamais accès formellement à l'information. Cet accès se faisait de manière informelle, de bouche à oreille. Dans la FC A4, ce n'est qu'après le soulèvement de la communauté que la transparence s'est améliorée et que les documents de la FC ont commencé à être accessibles. Le nombre de participants aux réunions a alors augmenté. Des négociations étaient organisées en privé entre les gestionnaires de la FC et certains groupes exclus de la FC. Une femme (F1) a affirmé qu'après négociation ces gestionnaires rapportaient les accords de la réunion privée et les avantages accordés à certains groupes exclus à toute la communauté et cela entraînait le soulèvement de nouveaux groupes.

\section{Légitimité}

Les acteurs tournés vers la communauté ont identifié les besoins des communautés et joué un rôle dans l'organisation des bureaux de gestion des FC. Pour eux, faire participer toutes les couches sociales représentait une exigence de la loi des forêts. Le choix des gestionnaires se faisait par vote, en présence des membres des communautés, accordant ainsi à ces acteurs la légitimité de gouverner. Dans la FC A1, par exemple, l'ONG internationale et son ONG locale partenaire ont organisé une élection pour choisir les gestionnaires.

Dans les FC initiées par les acteurs tournés vers la ressource ligneuse, le choix des gestionnaires de la FC était sous la responsabilité des chefs traditionnels, des leaders communautaires ou des élites. Les opérateurs forestiers privés finançaient la création des FC sans s'impliquer dans la désignation des gestionnaires. Lorsque les élites initiaient une $\mathrm{FC}$, elles procédaient par cooptation pour en désigner les gestionnaires, en fonction de leurs relations privées avec certaines personnes de la communauté. Les membres des communautés (femmes et jeunes) n'étaient pas d'accord, mais ne pouvaient s'opposer de peur d'être brimés.

\section{Équité}

Dans les FC initiées par les acteurs tournés vers la communauté et dans la FC spécifiquement initiée par les chefs traditionnels, les projets ont généré de meilleures retombées sociales. Les revenus générés permettaient des réalisations telles que la construction de foyers communautaires et l'achat de tôle (pour fabriquer des toits de maisons) et de partager des denrées alimentaires avec les communautés. Cependant, l'équité dans le choix des projets était mise en doute. Dans la FC A1, par exemple, après la première exploitation en 2017, les gestionnaires de la FC avaient décidé d'offrir une maison à onze de ses membres. Les bénéficiaires étaient les fils des fondateurs de la FC décédés, en guise de reconnaissance pour leurs sacrifices, les gestionnaires actuels et le plus âgé du village. Les communautés estimaient que le partage n'était pas équitable et que les revenus de la FC devaient bénéficier à tout le village.

Dans les FC initiées par les opérateurs forestiers privés et les élites, la gestion des revenus était inconnue des membres de la communauté. L'argent servait uniquement à l'achat et au partage des denrées alimentaires.

\section{Intégration}

Les acteurs tournés vers la communauté avaient le souci d'améliorer le bien-être dans les FC et cherchaient à coordonner les activités des différents acteurs pour en assurer l'intégration. Dans la FC A1, l'ONG internationale a créé de nouvelles activités pour adapter son appui aux besoins de la communauté. Ainsi, en plus des activités d'exploitation du bois, la collecte de PFNL était effectuée. L'Église et son ONG nationale partenaire n'avaient pas de fonds pour intégrer les besoins des communautés qui ne cadraient pas avec leur projet. 
Dans les FC initiées par les acteurs tournés vers la ressource, les gestionnaires étaient les seuls à définir ou à connaître la vision de la FC et les besoins de la communauté. Dans la FC A6, après le décès de l'ancien maire (élite), le gestionnaire (fils de l'élite) était devenu le seul à connaître la vision de la FC et gérait seul avec les opérateurs forestiers privés. Dans la FC A4, c'est après le soulèvement de la communauté que les gestionnaires ont commencé à intégrer les besoins de la communauté dans la FC.

\section{Imputabilité}

Dans les FC initiées par les acteurs tournés vers la communauté, la responsabilité de la gestion des contrats revenait à toute la communauté. Ces acteurs, par l'entremise d'accords avec les leaders de la communauté, développaient des activités d'exploitation forestière et de collecte de PFNL dont certains membres de la communauté étaient responsables. Ces acteurs vérifiaient les rapports de compte-rendu de performance des différents groupes. Cette reddition de comptes témoignait d'une imputabilité des acteurs.

Les gestionnaires des FC initiées par les élites et les opérateurs forestiers privés supervisaient les activités et le suivi des dossiers avec l'appui des opérateurs forestiers privés. Ces gestionnaires étaient responsables de la négociation des contrats avec les opérateurs forestiers privés. Les communautés ne recevaient aucun compte-rendu de la part des gestionnaires. Dans ces FC, les gestionnaires estimaient qu'ils n'avaient pas de véritable responsabilité étant donné que c'était l'opérateur forestier privé qui prenait en charge toutes les dépenses liées à l'obtention des documents et à l'achat du matériel pendant l'exploitation.

\section{Capacité}

Dans les FC initiées par les acteurs tournés vers la communauté, des formations sur la foresterie communautaire étaient dispensées aux gestionnaires pour améliorer leurs capacités. Une personne-ressource (P2) a affirmé que, lorsque les communautés n'assimilaient pas certaines formations, ces acteurs essayaient de corriger les erreurs.

Les gestionnaires des FC initiées par les acteurs tournés vers la ressource ligneuse n'avaient pas de véritables compétences en foresterie communautaire. Ces acteurs ne contribuaient pas à renforcer les capacités des communautés.

\section{Adaptabilité}

Les communautés ont eu des difficultés à s'adapter à la FC, soit à acquérir de nouvelles connaissances et à se réorganiser pour s'adapter aux changements. Malgré les contraintes de coûts et de cadre légal, les acteurs tournés vers la communauté ont pu amener les communautés à s'intéresser au concept de FC.

Dans les FC initiées par les opérateurs forestiers privés et les élites, les gestionnaires n'étaient pas favorables aux changements sans avoir obtenu, au préalable, l'accord des opérateurs forestiers. Les communautés affirmaient être méfiantes face aux situations changeantes dans le processus de FC. Les gestionnaires des FC affirmaient que les revenus et les motivations à s'adapter à la FC avaient diminué avec l'arrivée de la taxe d'abattage, appuyée par l'article 242 de la loi de finances au Cameroun n 2016/018 du 14 décembre $2016^{3}$. Cette taxe occasionnait des dépenses supplémentaires et, par conséquent, la baisse des revenus destinés à la réalisation des projets de développement des FC.

\section{Déterminants de la gouvernance des forêts communautaires}

L'augmentation d'un déterminant positif (+) ou l'atténuation d'un déterminant négatif (-) contribuent à la bonne gouvernance des FC (figure 3). Ces déterminants ont été regroupés en trois groupes: au sein de la communauté, au sein des acteurs d'appui ainsi qu'entre les communautés et les acteurs d'appui. Par exemple, au sein de la communauté, la cohésion sociale (+), en s'appuyant sur la réduction de l'exclusion (-), pourrait influencer les principes de participation et de légitimité. Au sein des acteurs d'appui, l'accès au marché des produits issus des FC (+) aurait un effet positif sur l'adaptation. Alors qu'entre les communautés et les acteurs d'appui l'assurance du respect des contrats (+) entre les opérateurs forestiers et les communautés renforcerait le principe d'imputabilité dans les FC.

\section{Échelle de gouvernance des forêts communautaires}

L'échelle de gouvernance des FC proposée ici se divise en trois niveaux : mal-gouvernance, vers la gouvernance et gouvernance (figure 4).

\section{Mal-gouvernance}

Le degré de mal-gouvernance se caractérise par l'ignorance ou l'exclusion et représente une FC monopolisée par un groupe restreint qui néglige le point de vue de la communauté. La communauté ignore qu'elle a une FC ou pense que la FC est la propriété de l'élite. La transparence, l'équité et la participation sont inexistantes. Un groupe restreint contrôle la FC et choisit les informations à communiquer aux autres groupes, qui sont exclus du processus décisionnel. L'information est à sens unique et ne tient pas compte du point de vue des communautés.

\section{Vers la gouvernance}

Au degré intermédiaire de gouvernance, les communautés ont l'assurance d'être représentées et consultées. Toutefois, les activités sont prévues à l'avance sans tenir compte du savoir local. Les opinions de la communauté sont sondées au moyen de concertations lors de rencontres d'échange entre les communautés et l'acteur d'appui. Cependant, les acteurs ont des revenus limités pour prendre en compte les besoins des communautés.

3. Loi $n^{\circ} 2016 / 018$ du 14 décembre 2016 portant sur les finances de la République du Cameroun pour l'exercice 2017. Ministère des Finances. République du Cameroun. 


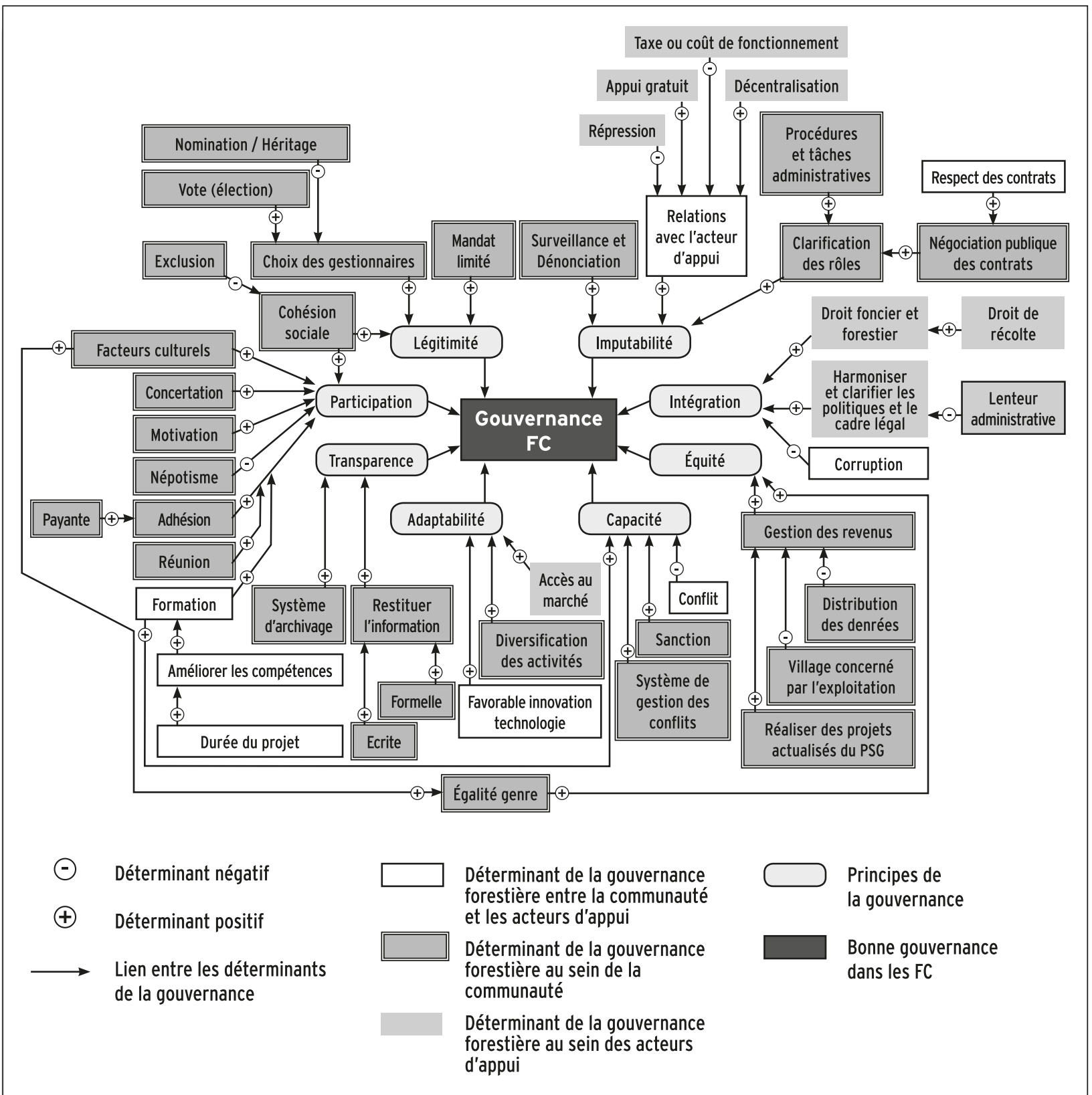

Figure 3.

Déterminants de la gouvernance des forêts communautaires

(FC) de l'Est-Cameroun (inspiré de Lockwood et al., 2010 et de Baynes et al., 2015).

\section{Gouvernance}

$\mathrm{Au}$ plus haut degré de gouvernance, la communauté contrôle la FC en partenariat avec l'acteur d'appui. Les acteurs tournés vers la communauté procèdent par négociation avec les communautés pour rechercher le consensus. Dans la FC A1, l'acteur d'appui et la communauté avaient presque le même niveau de pouvoir. L'acteur d'appui échangeait connaissances et expériences avec la communauté, mais il conservait le pouvoir de juger de la légitimité des propositions des communautés. Le niveau de gouvernance pourrait être encore plus élevé avec la dévolution. La dévolution permet aux communautés de maîtriser la FC pour assumer pleinement leur rôle. Les communautés sont habilitées par l'État à initier leurs propres règles de gestion selon leur savoir local de gestion forestière. Cependant, aucune des FC à l'étude n'a présenté un tel degré de gouvernance. L'atteinte de la dévolution dans les FC étudiées nécessite la prise en compte de tous les déterminants. La non-prise en compte d'un des déterminants pourrait entraîner l'échec de la dévolution et favoriser la confiscation de la FC par un groupe restreint. 


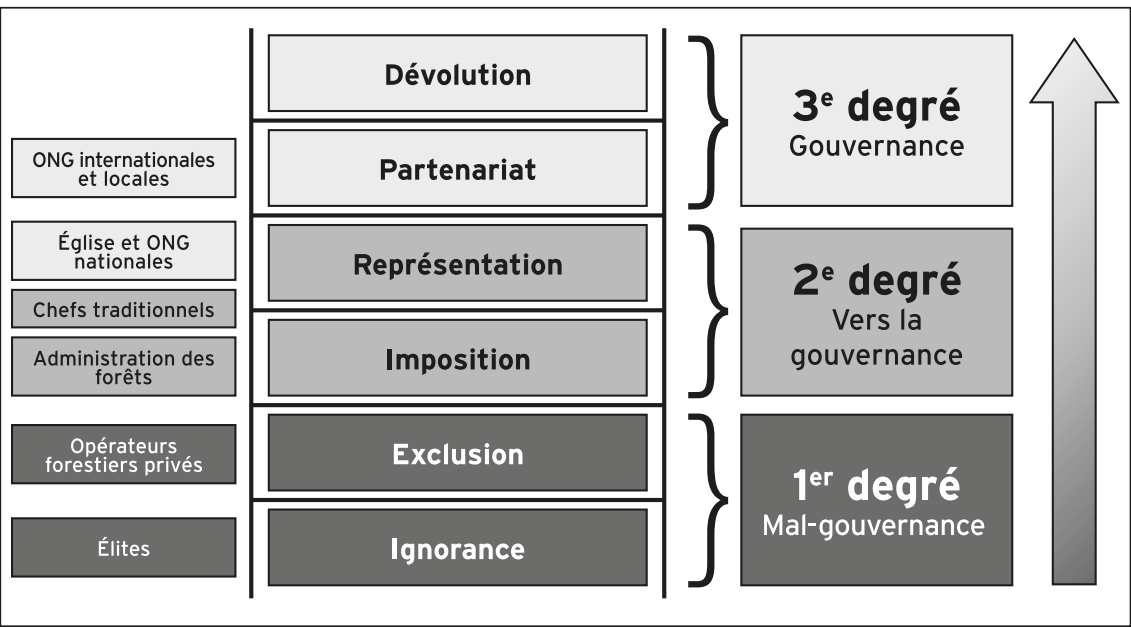

Figure 4.

Échelle de gouvernance des forêts communautaires (FC) de

l'Est-Cameroun (inspiré de Arnstein, 1969 et de Rodon, 2003).

légitimité et de participation concordent avec l'étude de Oyono (2004) selon laquelle les élites organiseraient des élections non démocratiques pour obtenir des postes dans les FC. Ces gestionnaires sont choisis à cause de leur lien avec les grands propriétaires fonciers (Oyono, 2005 ; Cuny, 2011). Dans les FC initiées par les acteurs tournés vers la communauté, le choix des gestionnaires ne tenait pas compte du droit foncier. Les FC étaient basées uniquement sur l'entité juridique reconnue par l'État et les clans ou lignages étaient ignorés dans la gestion des FC, contrairement aux forêts des communautés locales de la République démocratique du Congo, qui prennent en compte les leaders traditionnels (Vermeulen et Karsenty, 2017). Le modèle camerounais a fait naître une nouvelle classe de leaders politiques non issus du système de lignage traditionnel malgré

\section{Discussion}

\section{Acteurs d'appui à la création des forêts communautaires}

La cartographie des acteurs d'appui révèle que, dans les 12 FC étudiées, les communautés ont presque toujours eu l'appui des ONG, de l’Église, des élites ou des opérateurs forestiers privés pour s'engager dans le processus de création des FC. Seule la FC A3 a pu être créée par les communautés sous la seule impulsion des chefs traditionnels. Les études de Ezzine de Blas et al. (2009) et de Minang et al. (2019) au Cameroun ont en effet souligné que l'appui d'une élite, des opérateurs forestiers privés ou d'une ONG était nécessaire pour générer les revenus nécessaires à la création d'une FC. Diverses études, dont celles de Bigombe Logo (2007) et Lescuyer et al. (2016) au Cameroun et de McCarthy (2005) aux États-Unis, ont exploré le processus de création de FC et ont relevé qu'aucune communauté n'aurait pu obtenir une FC sans le soutien direct ou indirect d'acteurs d'appui. La nature du soutien diffère en fonction de ces acteurs. Les ONG représentent des banques de financement pour couvrir les coûts liés à la création des FC ou mènent des plaidoyers pour la révision des lois forestières, alors que les opérateurs forestiers privés apportent plutôt un soutien financier remboursable (Burnham, 2000 ; Djeumo, 2001 ; Pokharel et al., 2007 ; Ezzine de Blas et al., 2009 ; Minang et al., 2019).

\section{Perception de la gouvernance forestière}

La perception par les communautés de la gouvernance des FC variait en fonction des acteurs d'appui (tableau III). Les acteurs tournés vers la communauté avaient davantage tendance à respecter les principes de gouvernance.

Dans les FC initiées par les acteurs tournés vers la ressource ligneuse, la désignation des gestionnaires de la FC se faisait par cooptation ou par nomination de manière à assurer la représentation des grandes familles. Ces modes de les frustrations observées (Milol, 2007). Ce modèle dépossédait les chefs de lignage et les grands propriétaires fonciers de leur fonction de représentation de la communauté et remettait en cause l'organisation politique des villages.

Les acteurs tournés vers la communauté s'impliquaient dans l'élection des gestionnaires de la FC et exigeaient la représentation de toutes les couches sociales au comité de gestion de la FC. Cette organisation des élections et les échanges informels constituaient aussi un moyen de participation du public (Teitelbaum et al., 2006). Des recherches antérieures ont souligné que les ONG affectaient la hiérarchie sociale en choisissant de façon démocratique les gestionnaires des FC (Pokharel et al., 2007 ; Afroz et al., 2016). Une étude de Blomley et al. (2008) en Tanzanie signale que la participation des communautés a un impact positif sur l'état de la forêt. Cependant, cette participation est une chose et l'influence réelle en est une autre et exige souvent des changements substantiels dans les pratiques (Thoms, 2008 ; Lockwood et al., 2010). Les résultats montrent que l'implication des acteurs tournés vers la communauté permet de développer la légitimité des gestionnaires de la FC et d'améliorer la gouvernance.

Les principes de transparence, d'équité, d’imputabilité et de capacité étaient mieux respectés lorsque l'appui venait des acteurs tournés vers la communauté ainsi que dans la FC spécifiquement initiée par des chefs traditionnels. Les communautés avaient alors accès à l'information, aux documents et le processus de prise de décision ou de gestion des revenus de la FC n'était pas monopolisé par un groupe restreint. Ces résultats confirment ceux de Persha et Andersson (2014) qui soutiennent qu'en présence des ONG et des autres acteurs tournés vers la communauté la transparence est plus élevée et la captation de l'information ou des revenus de la FC par l'élite est réduite. Pour Gross-Camp (2017), même si la plupart des communautés avaient accès à l'information, elles n'étaient pas capables d'exercer une influence sur la prise de décision de la FC. Dans les FC initiées par les 
élites et les opérateurs forestiers privés, les communautés n'avaient pas accès à l'information. Le partage des revenus n'était pas équitable. Les études de Brown et Schreckenberg (2001) et Yadav et al. (2015) signalent que le partage des denrées était basé sur le nombre de ménages et non sur le nombre de personnes dans le ménage. Dans les FC étudiées, la présence des acteurs tournés vers la communauté et des chefs traditionnels constituait un facteur important pour améliorer la transparence, l'équité, la capacité et l'imputabilité.

Finalement, l'adaptabilité et l'intégration étaient plus grandes dans les FC initiées par l'ONG internationale et les chefs traditionnels. Ezzine de Blas et al. (2009) notent que la tendance à l'intégration augmente en présence d'un soutien externe. L'exception est que les communautés sans soutien extérieur peuvent atteindre un niveau d'intégration et d'adaptabilité plus élevé que les FC qui ne reçoivent que le soutien de l'administration des forêts (Ezzine de Blas et al., 2009 ; Ostrom, 2010). Cette exception est à rapprocher de celle de la FC A3 où les chefs traditionnels avaient pu améliorer l'intégration et l'adaptabilité de la FC dans la communauté.

Vers une « bonne gouvernance » : le rôle des déterminants

Les résultats de cette étude montrent que la cohésion sociale, la gestion des conflits, le partage équitable, le respect des contrats et l'accès au marché constituent des déterminants permettant le renforcement des principes de bonne gouvernance que sont la participation, la transparence, la légitimité, l'équité, l'intégration, l'imputabilité, la capacité et l'adaptabilité. Ces résultats concordent avec ceux de Ostrom (2010), Baynes et al. (2015, 2016) et Gilmour (2016), qui ont observé que le processus équitable de prise de décision, le contrôle de l'accès aux ressources, l'amélioration du cadre réglementaire, l'accès aux marchés, la réduction des inégalités sociales et la prise en compte par l'État central des pratiques locales constituaient des déterminants de la bonne gouvernance forestière. La cohésion entre les principes de gouvernance forestière et leurs déterminants a permis de concevoir un diagramme relationnel permettant d'identifier les actions correctives à entreprendre pour mieux tenir compte des besoins des communautés afin d'atteindre les échelons les plus élevés de l'échelle de la gouvernance. Cette échelle, comme celle de Baynes et al. (2015), identifie les déterminants négatifs à atténuer et positifs à augmenter au sein de la communauté, parmi les acteurs d'appui, ainsi qu'entre les communautés et les acteurs d'appui. Les résultats présentés ici montrent que cette cohésion est nécessaire pour atteindre une réelle dévolution sur l'échelle de gouvernance des FC.

\section{Échelle de la gouvernance forestière : un outil diagnostic}

L'étude de 12 FC au Cameroun a permis de proposer une échelle de gouvernance basée sur les travaux de Arnstein (1969) et de Rodon (2003). L'accès à l'information, l'amélioration de la cohésion sociale et la réduction des conflits ne permettraient pas à eux seuls de garantir le respect des principes de gouvernance dans les FC et encore moins l'atteinte de la dévolution. Arnstein (1969) signale que l'accès à l'information ne garantit pas la participation. Seule la prise en compte de tous les déterminants de la gouvernance permettrait la dévolution aux communautés de la gestion des FC. Cependant, en transférant une part plus grande du pouvoir de décision mal contrôlé aux acteurs et institutions locaux, la dévolution pourrait conduire à un risque plus élevé de captation par l'élite (Persha et Andersson, 2014). Une approche ascendante qui prend en compte les besoins, les connaissances et les réalités des communautés peut conduire à des systèmes de soutien mieux conçus que ceux mis en place de l'extérieur (Lescuyer et al., 2019).

\section{Conclusion}

Des 12 forêts communautaires (FC) étudiées, seule la FC A3 a été créée par des chefs traditionnels. Ce fait démontre l'importance du soutien des acteurs d'appui lors de la création des FC. Dans les FC étudiées, la nature du soutien différait en fonction des acteurs d'appui. Les acteurs tournés vers la communauté fournissent un soutien financier non remboursable lors de la création des FC, alors que les acteurs tournés vers la ressource ligneuse apportent plutôt un soutien financier remboursable.

Les communautés avaient une perception plus positive de la gouvernance dans les FC initiées avec l'appui d'acteurs tournés vers la communauté. En effet, ces derniers contribuaient à améliorer le respect des principes de participation, d'intégration, de capacité, et de transparence. Les acteurs tournés vers la communauté s'impliquaient dans l'élection des gestionnaires de la FC et exigeaient la représentation de toutes les couches sociales au comité de gestion de la FC, sans tenir compte de l'organisation politique lignagère des villages.

Les principes de légitimité, d'équité, d'imputabilité et d'adaptabilité étaient plus difficilement respectés dans les FC étudiées. La prise en compte de la cohésion entre les principes de gouvernance forestière et leurs déterminants est nécessaire pour atteindre la dévolution sur l'échelle de la gouvernance des FC. Le non-respect d'un principe ou la non-prise en compte d'un déterminant ainsi que des interactions entre eux peuvent contribuer à l'échec de l'atteinte de la dévolution. L'échelle de gouvernance proposée pourra servir d'outil de diagnostic des FC afin d'identifier les actions correctives à prendre pour mieux tenir compte des besoins des communautés.

\section{Remerciements}

Nous remercions le Bureau international de l'Université Laval pour le financement de la collecte de données ; les communautés des forêts communautaires de l'Est-Cameroun et les étudiants de la Faculté d'agronomie et des sciences agricoles de l'Université de Dschang pour la collecte de données ; le personnel graphiste de la Faculté de foresterie, de géographie et de géomatique de l'Université Laval pour la réalisation des figures.

Il n'existe aucun conflit d'intérêts entre les auteurs et les acteurs d'appui qui ont soutenu, de près ou de loin, les 12 FC étudiées, ni avec les FC elles-mêmes. L'absence de mention des noms des FC étudiées et des acteurs d'appui découle du respect des règles édictées par les Comités d'éthique de la recherche avec des êtres humains de l'Université Laval. 


\section{Références bibliographiques}

Afroz S., Cramb R., Grünbühel C., 2016. Ideals and institutions : Systemic reasons for the failure of a social forestry program in south-west Bangladesh. Geoforum, 77: 161-173. https://doi. org/10.1016/i.geoforum.2016.11.001

Alemagi D., Kozak R. A., 2010. Illegal logging in Cameroon: Causes and the path forward. Forest Policy and Economics, 12 (8): 554-561. https://doi.org/10.1016/j.forpol.2010.07.008

Arnstein S. R., 1969. A ladder of citizen participation. Journal of the American Planning Association, 35 (4): 216-224. http://dx.doi. org/10.1080/01944366908977225

Banque mondiale, 2009. Roots for good forest outcomes: An analytical framework for governance reforms. Washington, DC, USA, The World Bank, Agriculture and Rural Development Department, Report no. 49572-GLB, 64 p. http://siteresources.worldbank.org/INTARD/ Resources/forest_governance_combined_web_version.pdf

Baynes J., Herbohn J., Smith C., Fisher R., Bray D., 2015. Key factors which influence the success of community forestry in developing countries. Global Environmental Change, 35: 226-238. https://doi. org/10.1016/i.gloenvcha.2015.09.011

Baynes J., Herbohn J., Dressler W., 2016. Power relationships: Their effect on the governance of community forestry in the Philippines. Land Use Policy, 54: 169-176. https://doi.org/10.1016/i.landusepol.2016.01.008

Beaud J. P., 2009. L'échantillonnage. In : Gauthier B. (éd.). Recherche sociale, 5 é édition : De la problématique à la collecte des données. Québec, Canada, Presses de l'Université du Québec, 251-283. https://muse.jhu.edu/book/20328

Bigombe Logo P., 2007. Les régimes de la tenure forestière et leurs incidences sur la gestion des forêts et la lutte contre la pauvreté au Cameroun. Yaoundé, Cameroon, FAO, GRAPS, 31 p. http://www.fao. org/forestry/12717-09bdf38d000abba2b9e4d9c56e946b22e.pdf

Blomley T., Pfliegner K., Isango J., Zahabu E., Ahrends A., Burgess N., 2008. Seeing the wood for the trees: An assessment of the impact of participatory forest management on forest condition in Tanzania. Oryx, 42 (3): 380-391. https://doi.org/10.1017/ $\underline{\mathrm{s} 0030605308071433}$

Brown D., Schreckenberg K., 2001. Foresterie communautaire : Relever le défi camerounais. Londres, Royaume-Uni, Réseau de foresterie pour le développement rural, DFID, FRR, ODI, document du réseau 25a, 21 p. https://www.odi.org/sites/odi.org.uk/files/ odi-assets/publications-opinion-files/1426.pdf

Brunner J., Ekoko F., 2000. La réforme de la politique forestière au Cameroun : Enjeux, bilan, perspectives. Washington, DC, USA, World Resources Institute, 25 p. https://carpe.umd.edu/sites/default/ files/documentsarchive/wri-cameroon_casestudy2000-french.pdf

Burnham P., 2000. Whose forest? Whose myth? Conceptualisations of Community Forests in Cameroon. In: Abramson A., Theodossopoulos D. (eds). Land, Law and Environment: Mythical Land, Legal Boundaries. London, United Kingdom, Pluto Press, 31-58. https://doi.org/10.2307/i.ctt18fs63f.4

CARFAD (Centre africain de recherches forestières appliquées et de développement), MINFOF (Ministère des Forêts et de la Faune), 2006. Bilan des acquis de la foresterie communautaire au Cameroun et définition de nouvelles orientations. Yaoundé, Cameroun, CARFAD, 114 p. http://awsassets.panda.org/downloads/txt6 rapportbilanacquisforesteriecommunautaire_20080422_pdf

Cuny P., 2011. État des lieux de la foresterie communautaire et communale au Cameroun. Wageningen, Pays-Bas, Tropenbos International Programme du bassin du Congo, $110 \mathrm{p}$.
Djeumo A., 2001. Développement des forêts communautaires au Cameroun : Genèse, situation actuelle et contraintes. Londres, Royaume-Uni, Réseau de foresterie pour le développement rural, DFID, FRR, ODI, document du réseau 25b, $17 \mathrm{p}$. https:// www.odi.org/sites/odi.org.uk/files/odi-assets/publications-opinion-files/1428.pdf

EzzinedeBlasD., RuizPérezM.,SayerJ.A.,LescuyerG., Nasi R., Karsenty A., 2009. External influences on and conditions for community logging management in Cameroon. World Development, 37 (2): 445-456. https://doi.org/10.1016/j.worlddev.2008.03.011

Geoffrion P., 2009. Le Groupe de discussion. In : Recherche sociale, $5^{e}$ édition: De la problématique à la collecte des données. Québec, Canada, Presses de l'Université du Québec, 391-414. https:// muse.jhu.edu/book/20328

Gilmour D., 2016. Forty years of community-based forestry: A review of its extent and effectiveness. Rome, Italy, FAO, Forestry Paper no. 176, 168 p. http://www.fao.org/3/a-i5415e.pdf

Gross-Camp N., 2017. Tanzania's community forests : their impact on human well-being and persistence in spite of the lack of benefit. Ecology and Society, 22 (1): 37. https://doi.org/10.5751/ es-09124-220137

Julve Larrubia C., Vandenhaute M., Vermeulen C., Castadot B., Ekodeck H., DelvingtW., 2007. Séduisante théorie, douloureuse pratique : la foresterie communautaire camerounaise en butte à sa propre législation. Parcs et Réserves, 62 (2) : 1824. https://www.semanticscholar.org/paper/S\%C3\%A9duisante-th\%C3\%A9orie\%2Cdouloureuse-pratique\%3A-la-en-\%C3\%A0-Larrubia-Vandenhaute/ e4b78dfee936e150157f2632b7d32c2442eeb9cc

Julve Larrubia C., Eckebil P. P., Saha N. N., Tchantchouang J. C., Kerkhofs B., Beauquin A., et al., 2013. Forêts communautaires camerounaises et plan d'action « Forest Law Enforcement, Governance and Trade " (FLEGT) : quel prix pour la légalité ? Bois et Forêts des Tropiques, 317 (3): 7180. https://doi.org/10.19182/ bft2013.317.a20526

Karsenty A., 2010. Le nouveau " grand jeu » économique en Afrique et l'avenir des réformes de la gouvernance du secteur forestier. In : German L. A., Karsenty A., Tiani A.-M. (éds). Gouverner les forêts africaines à l'ère de la mondialisation. Bogor, Indonésie, CIFOR, 85-102. http://www.cifor.org/publications/pdf files/Books/BGerman0101.pdf

Karsenty A., 2017. The World Bank's endeavours to reform the forest concessions' regime in Central Africa: Lessons from 25 years of efforts. International Forestry Review, 19 (S2): 64-79. https://doi. org/10.1505/146554817822295948

Kouna Eloundou C. G., 2012. Décentralisation forestière et gouvernance locale des forêts au Cameroun : Le cas des forêts communales et communautaires dans la région Est. Thèse de doctorat, Université du Maine, France, 351 p. https://tel.archives-ouvertes. fr/tel-00795695/document

Lescuyer G., Cerutti P. O., Tsanga R., 2016. Contributions of community and individual small-scale logging to sustainable timber management in Cameroon. International Forestry Review, 18 (S1): 40-51. https://doi.org/10.1505/146554816819683744

Lescuyer G., Kakundika T., Lubala I., Ekyamba I., Tsanga R., Cerutti P. O., 2019. Are community forests a viable model for the Democratic Republic of Congo ? Ecology and Society, 24 (1): 6. https://doi. org/10.5751/es-10672-240106

Lockwood M., Davidson J., Curtis A., Stratford E., Griffith R., 2010. Governance principles for natural resource management. Society and Natural Resources, 23 (10): 986-1001. https://doi. org/10.1080/08941920802178214

Massoi L., Norman A. S., 2009. Decentralisation by devolution in Tanzania: Reflections on community involvement in the planning process in Kizota Ward in Dodoma. Journal of Public Administration and Policy Research, 1 (7): 133-140. https://academicjournals.org/ article/article1379494818_Massoi\%20and\%20Norman.pdf 
McCarthy J., 2005. Devolution in the woods: Community forestry as hybrid neoliberalism. Environment and Planning, 37: 995-1014. https://doi.org/10.1068/a36266

Milol C. A., 2000. Gestion des forêts communautaires au Cameroun : Enjeux, stratégies de mise en place, conflits. Étude de cas. In : Bahuchet S., Bley D., Pagezy H., Vernazza-Licht N. (éds). L'homme et la forêt tropicale. Châteauneuf-de-Grasse, France, Éditions de Bergier, 487-499. http://www.ecologie-humaine.eu/fr/fichiers/ PubPFR FOR.htm

Milol C. A., 2007. Gouvernance et participation dans la gestion des ressources forestières au Cameroun : impacts inattendus sur les pratiques foncières. In : Eberhard C. (dir.). Enjeux fonciers et environnementaux. Dialogues afro-indiens. Pondichéry, Inde, Institut français de Pondichéry, 233-255. http://www.foncier-developpement.fr/publication/gouvernance-et-participation-dans-la-gestion-des-ressources-forestieres-au-cameroun-impacts-inattendus-sur-les-pratiques-foncieres/

Minang P. A., McCall M. K., Bressers H. T. A., 2019. Evolution of community forestry in Cameroon: an innovation ecosystems perspective. Ecology and Society, 24 (1): 113. https://doi.org/10.5751/ ES-10573-240101

Ongolo S., Brimont L., 2015. Sociétés civiles et gestion communautaire des forêts en Afrique : Leçons et perspectives pour la gouvernance environnementale. In : Friboulet J.-J., Brot J., Gérardin H. (dir.). Dynamiques des sociétés civiles en économies ouvertes. Études de cas et perspectives (Afrique de l'Ouest, Europe, Maghreb). Paris, France, Karthala, 197-220.

Ostrom E., 2010. Gouvernance des biens communs : pour une nouvelle approche des ressources naturelles. Louvain-la-Neuve, Belgique, De Boeck, 301 p.

Oyono P. R., 2004. One step forward, two steps back? Paradoxes of natural resources management decentralisation in Cameroon. Journal of Modern African Studies, 42 (1): 91-111. https://doi. org/10.1017/s0022278x03004488

Oyono P. R., 2005. Profiling local-level outcomes of environmental decentralizations: The case of Cameroon's forests in the Congo basin. The Journal of Environment and Development, 14 (3): 317 337. https://doi.org/10.1177/1070496505276552

Paillé P., Mucchielli A., 2012. L'analyse qualitative en sciences humaines et sociales. $4^{\mathrm{e}}$ édition. Malakoff, France, Armand Colin, 424 p. https://doi.org/10.3917/arco.paill.2012.01

Persha L., Andersson K., 2014. Elite capture risk and mitigation in decentralized forest governance regime. Global Environmental Change, 24: 265-276. https://doi.org/10.1016/i.gloenvcha.2013.12.005

PNDP (Programme national de développement participatif), 2012 Plan communal de développement de Nguelebok. Batouri, Cameroun, PNDP, 138 p. https://www.pndp.org/documents/PCD NGUELEBOK.pdf

Pokharel B. K., Branney P. J., Nurse M., Malla Y. B., 2007. Community forestry: Conserving forests, sustaining livelihoods and strengthening democracy. Journal of Forest and Livelihood, 6 (2): 819. https://www.nepjol.info/index.php/JFL/article/view/2321

Profor (Programme sur les forêts), FAO (Organisation des Nations unies pour l'alimentation et l'agriculture), 2011. Cadre pour l'évaluation et le suivi de la gouvernance forestière. Rome, Italie, FAO, 26 p. http://www.fao.org/3/i2227f/i2227f00.pdf

Ribot J. C., Agrawal A., Larson A. M., 2006. Recentralizing while decentralizing: How national governments reappropriate forest resources. World Development, 34 (11): 1864-1886. https://doi. org/10.1016/j.worlddev.2005.11.020

Rodon T., 2003. En partenariat avec l'État. Les expériences de cogestion des Autochtones du Canada. Québec, Canada, Presses de l'Université Laval, 315 p. https://doi.org/10.7202/012656ar

Samndong R. A., Vatn A., 2018. Competing tenures: Implications for REDD+ in the Democratic Republic of Congo. Forests, 9 (662): 118. https://doi.org/10.3390/f9110662
Teitelbaum S., Beckley T., Nadeau S., 2006. A national portrait of community forestry on public land in Canada. The Forestry Chronicle, 82 (3): 416-428. https://doi.org/10.5558/tfc82416-3

Thoms C. A., 2008. Community control of resources and the challenge of improving local livelihoods: A critical examination of community forestry in Nepal. Geoforum, 39 (3): 1452-1465. https:// doi.org/10.1016/i.geoforum.2008.01.006

Vermeulen C., Karsenty A., 2017. Towards a community-based concession model in the DRC. International Forestry Review, 19 (S2): 17. https://doi.org/10.1505/146554817822295894

Wright G. D., Andersson K. P., Gibson C. C., Evans T., 2016. Decentralization can help reduce deforestation when user groups engage with local government. Proceedings of the National Academy of Sciences, 113 (52): 14958-14963. https://doi.org/10.1073/ pnas.1610650114

Yadav B. D., Bigsby H., MacDonald I., 2015. The relative distribution: An alternative approach to evaluate the impact of community level forestry organisations on households. Land Use Policy, 42: 443449. https://doi.org/10.1016/i.landusepol.2014.08.019

\begin{tabular}{|c|c|}
\hline Rôle du contributeur & Noms des auteurs \\
\hline Conceptualisation & $\begin{array}{l}\text { R. Fapa Nanfack, N. Gélinas, } \\
\text { K. S. Bobo, H. Asselin, F. Hiol Hiol }\end{array}$ \\
\hline Gestion des données & $\begin{array}{l}\text { R. Fapa Nanfack, N. Gélinas, } \\
\text { K. S. Bobo, H. Asselin, F. Hiol Hiol, } \\
\text { C. Atole Ntatsoula }\end{array}$ \\
\hline Analyse formelle & R. Fapa Nanfack, N. Gélinas \\
\hline $\begin{array}{l}\text { Acquisition du } \\
\text { financement }\end{array}$ & N. Gélinas \\
\hline $\begin{array}{l}\text { Enquête et } \\
\text { investigation }\end{array}$ & R. Fapa Nanfack, C. Atole Ntatsoula \\
\hline Méthodologie & $\begin{array}{l}\text { R. Fapa Nanfack, N. Gélinas, } \\
\text { K. S. Bobo, H. Asselin, F. Hiol Hiol }\end{array}$ \\
\hline Gestion de projet & $\begin{array}{l}\text { R. Fapa Nanfack, N. Gélinas, } \\
\text { K. S. Bobo, H. Asselin, F. Hiol Hiol }\end{array}$ \\
\hline Ressources & $\begin{array}{l}\text { R. Fapa Nanfack, N. Gélinas, } \\
\text { K. S. Bobo, H. Asselin, F. Hiol Hiol }\end{array}$ \\
\hline Logiciels & R. Fapa Nanfack \\
\hline Supervision & $\begin{array}{l}\text { N. Gélinas, K. S. Bobo, H. Asselin, } \\
\text { F. Hiol Hiol }\end{array}$ \\
\hline Validation & $\begin{array}{l}\text { R. Fapa Nanfack, N. Gélinas, } \\
\text { K. S. Bobo, H. Asselin, F. Hiol Hiol }\end{array}$ \\
\hline Visualisation & R. Fapa Nanfack \\
\hline $\begin{array}{l}\text { Écriture - Préparation } \\
\text { de l'ébauche originale }\end{array}$ & R. Fapa Nanfack \\
\hline $\begin{array}{l}\text { Écriture - Révision et } \\
\text { édition }\end{array}$ & $\begin{array}{l}\text { R. Fapa Nanfack, N. Gélinas, } \\
\text { K. S. Bobo, H. Asselin, F. Hiol Hiol, } \\
\text { C. Atole Ntatsoula }\end{array}$ \\
\hline
\end{tabular}

Bois et Forêts des Tropiques - Revue scientifique du Cirad

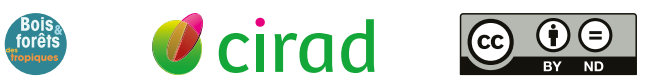

Cirad - Campus international de Baillarguet, 34398 Montpellier Cedex 5, France - Contact : bft@cirad.fr - ISSN : L-0006-579X 